\title{
Measuring anion binding at biomembrane interfaces
}

Xin Wu ${ }^{\mathrm{a}}$, Patrick Wang ${ }^{\mathrm{a}}$, William Lewis ${ }^{\mathrm{a}}$, Yun-Bao Jiang ${ }^{\mathrm{b}}$, and Philip A. Gale ${ }^{\mathrm{a},{ }^{*}}$

a School of Chemistry, The University of Sydney, NSW 2006, Australia

${ }^{\mathrm{b}}$ Department of Chemistry, College of Chemistry and Chemical Engineering, The MOE Key Laboratory of Spectrochemical Analysis and Instrumentation, and iChEM, Xiamen University, Xiamen 361005, China

*Corresponding author. E-mail address: philip.gale@sydney.edu.au

Abstract: Understanding non-covalent molecular recognition events at biomembrane interfaces is important in biological, medicinal, and materials chemistry research. ${ }^{1}$ Despite the crucial regulatory roles of anion binding/transport processes at biomembranes, no information is available regarding how strongly anions can bind to naturally occurring or synthetic receptors in lipid bilayer environments compared to their well-established behaviour in solutions. ${ }^{2}$ To bridge this knowledge gap, we synthesised a flat macrocycle that possesses a record aqueous $\mathrm{SO}_{4}{ }^{2-}$ affinity among neutral receptors and exploited its unique fluorescence response at interfaces. We show that the determinants of anion binding are extraordinarily different in organic solvents and in lipid bilayers. The high charge density of dihydrogen phosphate and chloride ions prevails in DMSO, however in lipids they fail to bind the macrocycle. Perchlorate and iodide hardly bind in DMSO but show significant affinities for the macrocycle in lipids. Our results demonstrate a surprisingly great advantage of large, charge-diffuse anions to bind to a lipid-embedded synthetic receptor mainly attributed to their higher polarisabilities and deeper penetration into the bilayer, beyond the common knowledge of dehydration energygoverned selectivity. The elucidation of these principles enhances our understanding of biological anion recognition functions in membranes and guides the design of ionophores and molecular machines operating at biomembrane interfaces.

The electrostatic and chemical properties of lipid bilayers have profound impacts on chemical reactions ${ }^{3}$ and non-covalent molecular recognition events ${ }^{4}$ occurring at water/membrane interfaces. Despite advances in anion receptor chemistry ${ }^{5}$ and the knowledge that lipophilic anion receptors can carry anions across cell membranes, ${ }^{6}$ lipid bilayers remain an unexplored territory for fundamental anion binding studies. While in bulk solvents, anion binding affinities were usually determined by NMR titrations, ${ }^{2}$ technical difficulties including poor solubilities, signal broadening, and weak anion affinities have so far impeded the application of NMR techniques to elucidating anion binding in native lipid bilayer environments.

We here report a $D_{3 h}$-symmeric tris(carbazole-urea) macrocycle 1 (Fig.1a) and the finding that the fluorescence properties of the macrocycle allow anion binding studies to be performed in lipid bilayer membranes. Macrocycle 1 has a $3.5 \AA$ cavity composed of a well pre-organised array of nine strong $\mathrm{NH}$ hydrogen bond donors pointing inwards. This ensures interactions with large anions such as $\mathrm{SO}_{4}{ }^{2-}$ to be sufficiently strong and measurable in extremely competitive lipid bilayer environments (vide infra).

While the structure of trimeric macrocycle 1 was initially proposed in a theoretical study in $2017,{ }^{7}$ no synthetic progress was made despite the success with the dimeric ${ }^{8}$ and the tetrameric ${ }^{9}$ analogues. We have developed a remarkably simple one-pot $\mathrm{SO}_{4}{ }^{2-}$-templated synthesis to access the trimeric macrocycle 1 (Fig.1a). The crystal structure of $1-\mathrm{SO}_{4}{ }^{2-}$ (Fig.1b) shows a $D_{3 \mathrm{~d}}$-symmeric complex with three oxygen atoms of the central bound $\mathrm{SO}_{4}{ }^{2-}$ ion interacting with all urea motifs of 1 via six short 
$\mathrm{NH} \cdots \mathrm{O}$ contacts of $2.01 \AA$ and with carbazole $\mathrm{NHs}$ with longer $\mathrm{NH} \cdots \mathrm{O}$ contacts of $2.61 \AA$. The macrocycle was slightly buckled (Fig.1b right, dihedral angles between carbazole units measured to be $171.91^{\circ}$ ) to accommodate the large tetrahedral $\mathrm{SO}_{4}{ }^{2-}$ ion.

(a)

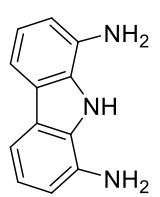

Triphosgene,

$\mathrm{NaHCO}_{3}$, $\mathrm{Na}_{2} \mathrm{SO}_{4}$

$\mathrm{EtOAc} / \mathrm{H}_{2} \mathrm{O}$ $30^{\circ} \mathrm{C}$

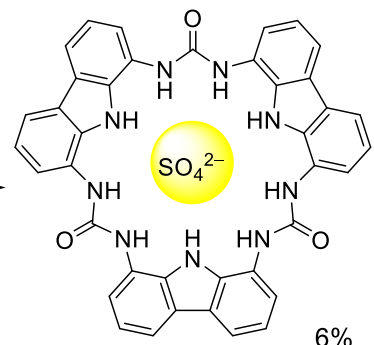

1

$6 \%$ (c)

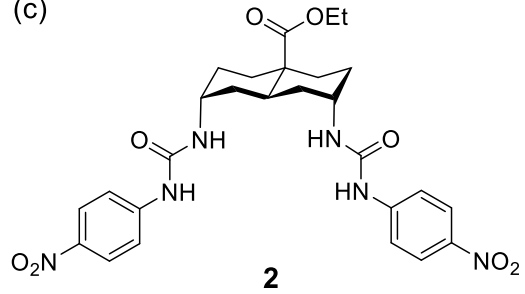

(b)
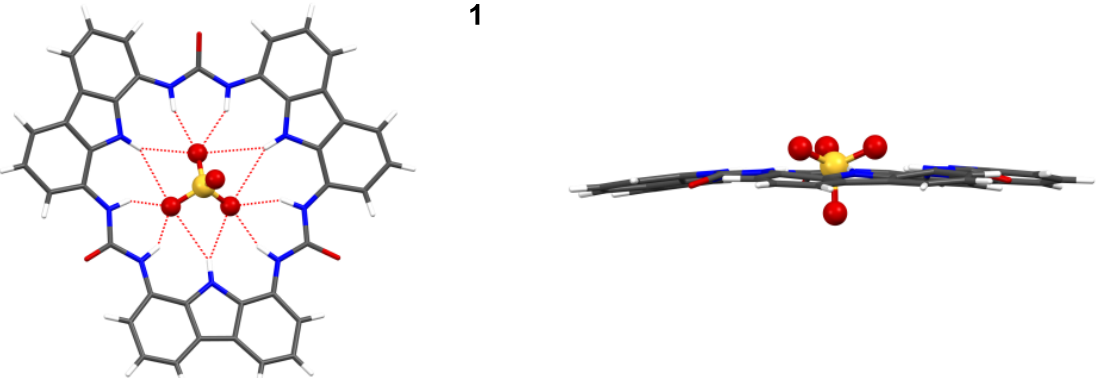

Fig.1 (a) Synthesis of macrocycle 1. The $\mathrm{SO}_{4}{ }^{2-}$ template could be removed by EtOAc/ $\mathrm{H}_{2} \mathrm{O}$ extraction, which, however, led to partial degradation of the macrocycle. The free macrocycle with $80-90 \%$ purity was used in ${ }^{1} \mathrm{H}$ NMR titrations in DMSO- $d_{6} / 0.5 \% \mathrm{H}_{2} \mathrm{O}$. The pure $\mathrm{SO}_{4}{ }^{2-}$ complex was used in fluorescence studies in water where the complex completely dissociated at $50 \mathrm{nM}$ due to the competitive aqueous conditions. (b) Crystal structure of $1-\mathrm{SO}_{4}{ }^{2-}$ complex (CCDC: 2128483 ) with the counterion and disorders omitted. (c) Reference bis-urea anion receptor $\mathbf{2}$.

We conducted ${ }^{1} \mathrm{H}$ NMR titrations of 1 with tetrabutylammonium $\left(\mathrm{TBA}^{+}\right)$salts of $\mathrm{SO}_{4}{ }^{2-}, \mathrm{H}_{2} \mathrm{PO}_{4}{ }^{-}, \mathrm{Cl}^{-}, \mathrm{Br}^{-}$, $\mathrm{NO}_{3}{ }^{-}, \mathrm{I}^{-}$and $\mathrm{ClO}_{4}{ }^{-}$in DMSO-$d_{6} / 0.5 \% \mathrm{H}_{2} \mathrm{O}$ (Table 1 ). Macrocycle 1 has an exceptionally strong $\mathrm{SO}_{4}{ }^{2-}$ affinity in the sub-nanomolar range, which necessitates the use of a $\mathrm{BaSO}_{4}$ precipitation method for quantification (SI). For monovalent anions, the anion binding affinity decreases in the order of $\mathrm{H}_{2} \mathrm{PO}_{4}^{-}>$ $\mathrm{Cl}^{-}>\mathrm{NO}_{3}^{-}>\mathrm{Br}^{-}>\mathrm{I}^{-}>\mathrm{ClO}_{4}^{-}$. Compared with Davis's acyclic bis-urea 2 (Fig.1c), ${ }^{10}$ the macrocyclic receptor has a modest 1-2 fold affinity enhancement for $\mathrm{Cl}^{-}, \mathrm{Br}^{-}$and $\mathrm{I}^{-}$, but a significant 33-fold enhancement for $\mathrm{NO}_{3}{ }^{-}$. Geometrical optimisations of the anion complexes suggest that $\mathbf{1}$ has a perfect size and shape complementary fit for $\mathrm{NO}_{3}{ }^{-}$resulting in a perfectly flat and $D_{3 h}$-symmeric complex (Fig.S3b). ${ }^{7}$ By contrast, the macrocyclic cavity is slightly too large for $\mathrm{I}^{-}$(Fig.S3c) and consequently much too large for $\mathrm{Br}^{-}$(Fig.S3d) and $\mathrm{Cl}^{-}$(Fig.S3e). It should be noted here that without using $\mathbf{2}$ as a reference receptor for comparison, the macrocycle's structural preference for $\mathrm{NO}_{3}{ }^{-}$would have been blurred in the binding constant data in DMSO, as charge-dense monovalent anions $\mathrm{H}_{2} \mathrm{PO}_{4}{ }^{-}$and $\mathrm{Cl}^{-}$have greater binding affinities than $\mathrm{NO}_{3}{ }^{-}$which only narrowly edges out $\mathrm{Br}^{-}$. Thus, in DMSO where anions hardly need to dehydrate to bind, the anion binding affinities are dominated by strength of electrostatic interactions leading to favourable binding of charge-dense anions. 
Table 1 Anion binding and transmembrane anion transport properties of $\mathbf{1}$, along with literature values of anion hydration free energies and anion binding properties of $\mathbf{2}$ and PC vesicles. Errors present SD from at least two experiments.

\begin{tabular}{|c|c|c|c|c|c|c|c|}
\hline \multirow{3}{*}{ Anion } & \multirow{3}{*}{ 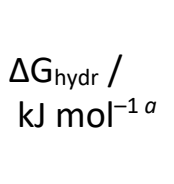 } & \multicolumn{5}{|c|}{ Association constant $K_{\mathrm{a}} / \mathrm{M}^{-1}$} & \multirow{3}{*}{$\begin{array}{l}\text { Transport } \\
\text { rate / anions } \\
\mathrm{s}^{-1} \text { carrier }^{-1 h}\end{array}$} \\
\hline & & \multicolumn{2}{|c|}{ in DMSO- $d_{6} / 0.5 \% \mathrm{H}_{2} \mathrm{O}$} & $\begin{array}{l}\text { in C12E8 } \\
\text { micelles }^{d}\end{array}$ & $\begin{array}{l}\text { In POPC } \\
\text { vesicles }^{d}\end{array}$ & PC & \\
\hline & & 1 & $2^{c}$ & 1 & 1 & & \\
\hline $\mathrm{SO}_{4}^{2-}$ & -1376 & $(7.4 \pm 1.1) \times 10^{9 b}$ & $>10^{5}$ & $54000 \pm 3000$ & $370 \pm 10^{e}$ & n.d. & $0.084 \pm 0.012$ \\
\hline $\mathrm{H}_{2} \mathrm{PO}_{4}^{-}$ & -473 & $>10^{5}$ & 46000 & $140 \pm 20$ & $<1$ & n.d. & $0.031 \pm 0.010$ \\
\hline $\mathrm{Cl}^{-}$ & -344 & $2000 \pm 100$ & 670 & $19 \pm 1^{e}$ & $<1$ & $0.2^{f}$ & $0.082 \pm 0.016$ \\
\hline $\mathrm{Br}^{-}$ & -318 & $200 \pm 10$ & 70 & $29 \pm 1^{e}$ & $2.6 \pm 0.6^{e}$ & $2^{f}$ & $0.097 \pm 0.018$ \\
\hline $\mathrm{NO}_{3}^{-}$ & -286 & $340 \pm 10$ & 10 & $210 \pm 10^{e}$ & $24 \pm 4^{e}$ & $2.8^{f}$ & $2.1 \pm 0.1$ \\
\hline $\mathrm{I}^{-}$ & -280 & $6.1 \pm 0.6$ & 3 & $200 \pm 10^{e}$ & $24 \pm 2^{e}$ & $32^{g}$ & $2.0 \pm 0.4$ \\
\hline $\mathrm{ClO}_{4}^{-}$ & -229 & $<1$ & n.d. & $32 \pm 1^{e}$ & $45 \pm 9^{e}$ & $115^{g}$ & $0.83 \pm 0.10$ \\
\hline
\end{tabular}

${ }^{a}$ Compiled by Marcus ${ }^{11} .{ }^{b}$ Determined using a $\mathrm{BaSO}_{4}$ precipitation method. ${ }^{c}$ Reported by Jurček et al. ${ }^{10}$ ${ }^{d}$ Surface potential effects corrected. ${ }^{e}$ Ionic strength fixed at 0.2. ${ }^{f}$ Reported by Tatulian, using egg PC vesicles. ${ }^{12}{ }^{g}$ Reported by Rydall and Macdonald, using POPC vesicles. ${ }^{13}{ }^{h}$ Determined at an anion concentration of $20 \mathrm{mM}$.

To evaluate the anion binding strength of $\mathbf{1}$ in water, we next switched the medium from DMSO to non-ionic octaethyleneglycol monododecyl (C12E8, Fig.2b) micelles dispersed in water, ${ }^{14}$ as neither 1 nor its anion complex is soluble in pure water. Gratifyingly, 1 can be solubilised at sub- $\mu \mathrm{M}$ concentrations in C12E8 micelles and demonstrates a strong fluorescence enhancement response to $\mathrm{SO}_{4}{ }^{2-}$ (Fig.S16), giving a remarkably high $\mathrm{SO}_{4}{ }^{2-}$ binding constant of $5.4 \times 10^{4} \mathrm{M}^{-1}$, which is $>20$ times greater than a bis-cyclopeptide, ${ }^{15}$ previously the highest affinity neutral $\mathrm{SO}_{4}{ }^{2-}$ receptor in pure water. ${ }^{16}$

Under water/C12E8 biphasic conditions, $\mathrm{H}_{2} \mathrm{PO}_{4}{ }^{-}$induced a fluorescence response similar to that of $\mathrm{SO}_{4}{ }^{2-}$ (Fig.S20), giving a modest $\mathrm{H}_{2} \mathrm{PO}_{4}^{-}$affinity of $140 \mathrm{M}^{-1}$. Other anions produced either negligible fluorescence responses $\left(\mathrm{Cl}^{-}\right)$or fluorescence quenching responses $\left(\mathrm{NO}_{3}{ }^{-}, \mathrm{I}^{-}\right.$and $\left.\mathrm{ClO}_{4}{ }^{-}\right)$partly attributable to a dynamic quenching mechanism (Fig.S22), rendering direct fluorescence titrations unfeasible. Instead, we conducted competitive titrations of $\mathrm{SO}_{4}{ }^{2-}$ in the presence of these anions and calculated the affinities based on attenuation of $\mathrm{SO}_{4}{ }^{2-}$ affinity using a competitive binding model ( $\mathrm{SI}$ ). In these analyses, it was necessary to correct the binding constant values against Boltzmann factors as anions adsorb to micellar surfaces ${ }^{17}$ leading to a negative surface potential (estimated by electrophoretic zeta potential measurements) which then reduces the $\mathrm{SO}_{4}{ }^{2-}$ concentration at the surface by a Boltzmann factor compared with in the bulk solution.

Table 1 demonstrates a modified anion selectivity in water/C12E8 compared with in DMSO, despite $\mathrm{SO}_{4}{ }^{2-}$ remaining as the most strongly bound anion. While $\mathrm{H}_{2} \mathrm{PO}_{4}{ }^{-}, \mathrm{Cl}^{-}$and $\mathrm{NO}_{3}{ }^{-}$are the top-3 strongest binding monovalent anions in DMSO, $\mathrm{Cl}^{-}$drops out of this group in the water/C12E8 system and is replaced by $\mathrm{I}^{-} \cdot \mathrm{H}_{2} \mathrm{PO}_{4}{ }^{-}$remains in top-3 but no longer has a huge advantage seen in DMSO. We rationalise the binding data on the basis of anion solvation free energies and dielectric constants of the media (Fig.2). The interfacial dielectric constant of C12E8 micelles was estimated to be $\sim 35,{ }^{18}$ which is similar to that of MeCN and lower than DMSO, and therefore anion binding should not be weakened solely based on medium polarity considerations. Here the diminished affinities of chargedense $\mathrm{SO}_{4}{ }^{2-}, \mathrm{H}_{2} \mathrm{PO}_{4}{ }^{-}$and $\mathrm{Cl}^{-}$anions, and the shift of anion selectivity towards more charge-diffuse $\mathrm{NO}_{3}{ }^{-}$ and $\mathrm{I}^{-}$anions are attributed to the heavier dehydration costs of the charge-dense than charge-diffuse anions. The augmented affinities of $\mathrm{I}^{-}$and $\mathrm{ClO}_{4}{ }^{-}$in water/C12E8 than in DMSO despite the anion 
dehydration cost in water/C12E8 can be rationalised by the high receptor desolvation cost in DMSO (see Fig.S10 for evidence of desolvation). ${ }^{16}$ We have also performed ${ }^{1} \mathrm{H}$ NMR of macrocycle $\mathbf{1}$ in water/C12E8 in the presence of anions (Fig.S30), in which the observation of resonances from anion complexes provided unambiguous evidence of binding of all tested anions in the biphasic system.

(a)

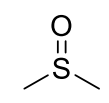

$\varepsilon=6.7$

Almost no dehydration requirement (DMSO- $d_{6} / 0.5 \% \mathrm{H}_{2} \mathrm{O}$ was used)

(c)

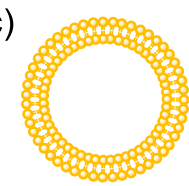<smiles>C[N+]1(C)CCOP(=O)(O)O1</smiles>

(b)

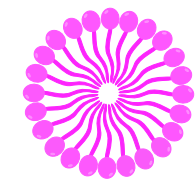

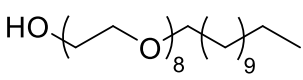

$\varepsilon$ (surface) $=35$

Anions need to dehydrate

Fig.2 Schematic representation of three media used for anion binding studies in this paper. (a) DMSO. (b) C12E8 micelles. (c) POPC vesicles.

Importantly, macrocycle 1 also demonstrated a fluorescence enhancement response to $\mathrm{SO}_{4}{ }^{2-}$ when incorporated at sub- $\mu \mathrm{M}$ concentrations in POPC (Fig.2C) vesicles suspended in water (Fig.S32). A further reduced $\mathrm{SO}_{4}{ }^{2-}$ affinity of $370 \mathrm{M}^{-1}$ was found for 1 in POPC vesicles compared with in C12E8 micelles (Table 1). This attenuation could be in part due to competitive receptor binding to the phosphate headgroup of POPC. ${ }^{19}$ To gain more evidence for this, we performed $\mathrm{SO}_{4}{ }^{2-}$ titrations of $\mathbf{1}$ in $10 \%$ and $20 \%$ POPC/C12E8 mixed micelles, which demonstrated 4.5 -fold and 9 -fold reduced $\mathrm{SO}_{4}{ }^{2-}$ affinity (Figs.S18,S19), respectively, compared with in pure C12E8 micelles. Further evidence was provided by the direct observation of ${ }^{1} \mathrm{H}$ NMR signals corresponding to the 1-POPC complex in POPC/C12E8 mixed micelles (Fig.S31).

Examination of binding constants of other anions in POPC vesicles (determined by competitive binding experiments with surface potential effects corrected, Table 1) have however revealed a trend that cannot be explained solely by competitive headgroup binding (which does not impact anion selectivity). Strikingly, while the divalent $\mathrm{SO}_{4}{ }^{2-}$ always remained the strongest binding anion, the topthree monovalent anion group changed again compared with in $\mathrm{C} 12 \mathrm{E} 8$ micelles, with $\mathrm{H}_{2} \mathrm{PO}_{4}{ }^{-}$being knocked out by $\mathrm{ClO}_{4}{ }^{-}$which joins $\mathrm{NO}_{3}{ }^{-}$and $\mathrm{I}^{-}$. In $\mathrm{POPC}$ vesicles, $\mathrm{H}_{2} \mathrm{PO}_{4}{ }^{-}$and $\mathrm{Cl}^{-}$no longer showed appreciable affinities $\left(K_{\mathrm{a}}<1 \mathrm{M}^{-1}\right)$. This trend also cannot be explained by anion dehydration cost alone, as anion binding in water/C12E8 and water/POPC systems have identical dehydration requirements. The dielectric property of the lipid bilayers, on the other hand, could provide a clue to the enhanced selectivity for charge-diffuse anions in POPC vesicles than in C12E8 micelles. Previously, two molecular dynamic simulation studies have estimated the dielectric constant of the zwitterionic headgroup region to be several times that of bulk water. ${ }^{20,21}$ Flood and coworkers have shown in a $\mathrm{Cl}^{-}$binding macrocycle that as the dielectric constant of the solvent increases, the energetic contribution of electrostatic interactions reduces while non-electrostatic induction and dispersion contributions start to dominate. ${ }^{22}$ We thus reason that should anion binding occur at the high electric constant headgroup region, charge-dense anions $\mathrm{SO}_{4}{ }^{2-}, \mathrm{H}_{2} \mathrm{PO}_{4}{ }^{-}$and $\mathrm{Cl}^{-}$that mainly rely on electrostatic interactions to bind would be disadvantaged over large charge-diffuse anions $\mathrm{ClO}_{4}{ }^{-}$and $\mathrm{I}^{-}$that have favourable induction and dispersion terms due to their polarizability. This effect would add to the chaotropic preference that arises from dehydration cost alone as we have already seen in C12E8 micelles.

Key information on the location of anion binding in POPC vesicles was then obtained by fluorescence penetration-depth studies using spin labelled lipids to quench the fluorescence of $\mathbf{1}$ at different locations (Figs.S46-S48). ${ }^{23}$ Without anions, the most probable location of macrocycle 1 was determined to be $19 \AA$ from the bilayer centre, corresponding to the headgroup region. This is 
consistent with the phosphate headgroup binding hypothesis. Macrocycle 1 remained at the headgroup region upon binding to $\mathrm{SO}_{4}{ }^{2-}$, but upon binding to $\mathrm{ClO}_{4}{ }^{-}$penetrated deeper (16 $\AA$ ) into the carbonyl/glycerol region with a lower dielectric constant of 3-4. ${ }^{20,21}$ Here we have confirmed that the binding of $\mathrm{SO}_{4}{ }^{2-}$ occurs at the high dielectric constant headgroup region, supporting the hypothesis that binding of charge-dense anions are subject to severe electrostatic screening which diminish their affinities (the $\mathrm{SO}_{4}{ }^{2-}$ selectivity persists, despite being much weaker than in DMSO and in C12E8 micelles, suggesting that very strong electrostatic interactions with multi-charged ions can still be impactful even with severe electrostatic screening). We have shown an additional benefit for chargediffuse anions such as $\mathrm{ClO}_{4}{ }^{-}$that their complexes can penetrate deeper into a more hydrophobic microenvironment where anion binding is enhanced.

It is of interest to compare the anion binding properties of macrocycle 1 in lipids against anion binding by lipids themselves. Lipid bilayers preferentially adsorb charge-diffuse anions and exhibit a Hofmeister selectivity pattern of $\mathrm{ClO}_{4}^{-}>\mathrm{I}^{-}>>\mathrm{NO}_{3}{ }^{-}>\mathrm{Br}^{-}>\mathrm{Cl}^{-}>\mathrm{H}_{2} \mathrm{PO}_{4}^{-}$(see also Table S3). ${ }^{12,13}$ Table 1 shows that macrocycle 1 binds $\mathrm{Br}^{-}, \mathrm{I}^{-}$and $\mathrm{ClO}_{4}{ }^{-}$with similar or weaker affinities than lipids, but binds $\mathrm{NO}_{3}{ }^{-} \sim 8$ times more strongly than lipids, again demonstrating the perfect size and shape matching of the macrocycle for $\mathrm{NO}_{3}{ }^{-}$(Fig. S3b). The ability of lipids to preferentially accumulate $\mathrm{ClO}_{4}^{-}$, on the other hand, likely compensates for enthalpic loss due to competitive lipid headgroup binding to 1 , leading to no attenuation of $\mathrm{ClO}_{4}{ }^{-}$affinity of $\mathbf{1}$ in POPC vesicles compared with in C12E8 micelles.

Further information came to light when we compare the abovementioned phenomena to cation binding to lipids and to the cation receptor/carrier valinomycin. The cation affinities of PC lipids among alkali metal cations from $\mathrm{Li}^{+}$to $\mathrm{Rb}^{+}$are very similar, ${ }^{24}$ while being far weaker than lipids binding chargediffuse anions. ${ }^{12,13}$ For valinomycin, although cation binding affinities dropped by several orders of magnitude when the medium switched from organic solvents to lipids, no drastic alteration of cation selectivity was found in lipids ${ }^{25}$ in contrast to the behaviour of "anti-valinomycin" 1 . As shown by previous theoretical investigations, the great polarisability of large charge-diffuse anions is central to their strong interfacial adsorption ${ }^{26,27}$ and in the cases of water/lipid interfaces, this then benefits binding of highly polarisable anions to an anion receptor embedded in lipids due to increased local anion concentrations. This effect is absent in cation binding because of the poor polarisabilities of cations.

Finally, we studied transmembrane anion transport ${ }^{28}$ properties of macrocycle $\mathbf{1}$ in POPC liposomes. Macrocycle 1 functions as an $\mathrm{H}^{+} /$anion $^{-}$symporter but not as an anion uniporter (Fig.S54) presumably due to the strong headgroup binding that inhibited transmembrane diffusion of the free receptor. ${ }^{19}$ An anion transport selectivity of $\mathrm{NO}_{3}{ }^{-} \approx \mathrm{I}^{-}>\mathrm{ClO}_{4}{ }^{-}>\mathrm{Br}^{-}>\mathrm{Cl}^{-} \approx \mathrm{SO}_{4}{ }^{2-}>\mathrm{H}_{2} \mathrm{PO}_{4}^{-}$was observed (Fig.S52,Table 1). Carrier-mediated ion transport rates depend both on the ion binding affinity and the rate of ion-carrier complex diffusion through the membrane ${ }^{29}$ the latter being clearly unfavourable for the doubly charged $\mathrm{SO}_{4}{ }^{2-}$. While $\mathrm{NO}_{3}{ }^{-}$and $\mathrm{I}^{-}$can be fully embedded into the macrocyclic plane (Figs.S3b,S3c), $\mathrm{ClO}_{4}{ }^{-}$has an exposed oxygen atom after binding to 1 (Fig.S3a), likely slowing down $\mathrm{ClO}_{4}{ }^{-}$ transport than $\mathrm{NO}_{3}{ }^{-}$and $\mathrm{I}^{-}$. In light of the $\mathrm{ClO}_{4}^{-}, \mathrm{I}^{-}>\mathrm{NO}_{3}{ }^{-}$transport selectivity commonly observed for structurally simple anion transporters following Hofmeister series, ${ }^{30}$ here the clear $\mathrm{NO}_{3}{ }^{-}>\mathrm{ClO}_{4}{ }^{-}$ transport selectivity of $\mathbf{1}$ again reflects macrocycle's structural fit for $\mathrm{NO}_{3}{ }^{-}$, which, however, is insufficient to confer a significant $\mathrm{NO}_{3}{ }^{-}>\mathrm{I}^{-}$selectivity due to the preference of the lipid environment for the more hydrophobic $\mathrm{I}^{-}$. This underscores the difficulty ${ }^{30}$ of overcoming the Hofmeister bias to facilitate selective membrane transport of hydrophilic anions such as $\mathrm{Cl}^{-}$which shows a deceptively strong affinity of $2000 \mathrm{M}^{-1}$ for 1 in DMSO.

In summary, we have gained access to the intricacies of anion binding at biomembrane interfaces taking advantages of an extremely strong $\mathrm{SO}_{4}{ }^{2-}$ binding macrocycle 1 showing fluorescence 
perturbation upon binding $\mathrm{SO}_{4}{ }^{2-}$ at interfaces. Across three tested media, we see a transition of the anion binding strength/selectivity of $\mathbf{1}$ from an electrostatics-dominated trend favouring kosmotropic, charge-dense anions in DMSO, to an electrostatics/dehydration co-governance in C12E8 micelles, and finally to a polarisability/penetration-depth/dehydration determined trend in POPC vesicles where chaotropic, charge-diffuse anions, in particular $\mathrm{ClO}_{4}{ }^{-}$, are surprisingly strongly favoured. In all media, we have seen the intrinsic size/shape matching selectivity of $\mathbf{1}$ for $\mathrm{NO}_{3}{ }^{-}$struggling to manifest itself amid the electrostatic, solvation and polarisability effects characteristic of the anions and medium conditions. We show a striking contrast to cation receptor chemistry in that anion polarisability plays a key role in determining anion binding strength/selectivity in lipids. Our insights are relevant to diverse research topics ranging from ion interactions with membrane-embedded proteins/peptides to drug delivery vehicles and synthetic receptors/transporters/assemblies functioning at biomembrane interfaces.

\section{Acknowledgement}

XW, PW, WL and PAG acknowledge and pay respect to the Gadigal people of the Eora Nation, the traditional owners of the land on which we research, teach and collaborate at the University of Sydney. We thank the Australian Research Council (DP210100039 and DE220101000), the National Natural Science Foundation of China (21820102006) and the University of Sydney for funding. We thank Prof Eric Ross (Gonzaga University) for helpful discussions, and Dr Nicholas Proschogo and Jakob D. Lane (The University of Sydney) for performing HRMS and ICP-MS.

1 Gonella, G. et al. Water at charged interfaces. Nat. Rev. Chem. 5, 466-485 (2021).

2 Busschaert, N., Caltagirone, C., Van Rossom, W. \& Gale, P. A. Applications of Supramolecular Anion Recognition. Chem. Rev. 115, 8038-8155 (2015).

3 Bondar, A.-N. \& Lemieux, M. J. Reactions at Biomembrane Interfaces. Chem. Rev. 119, 61626183 (2019).

4 Bickerton, L. E., Johnson, T. G., Kerckhoffs, A. \& Langton, M. J. Supramolecular chemistry in lipid bilayer membranes. Chem. Sci. 12, 11252-11274 (2021).

5 Chen, L., Berry, S. N., Wu, X., Howe, E. N. W. \& Gale, P. A. Advances in Anion Receptor Chemistry. Chem 6, 61-141 (2020).

6 Davis, J. T., Gale, P. A. \& Quesada, R. Advances in anion transport and supramolecular medicinal chemistry. Chem. Soc. Rev. 49, 6056-6086 (2020).

7 Bauzá, A., Frontera, A. \& Mooibroek, T. J. $\mathrm{NO}_{3}{ }^{-}$anions can act as Lewis acid in the solid state. Nat. Commun. 8, 14522 (2017).

8 Ahmed, N., Geronimo, I., Hwang, I.-C., Singh, N. J. \& Kim, K. S. cyclo-Bis(urea-3,6dichlorocarbazole) as a Chromogenic and Fluorogenic Receptor for Anions and a Selective Sensor of Zinc and Copper Cations. Chem. Eur. J. 17, 8542-8548 (2011).

9 Rüütel, A. et al. Design, synthesis and application of carbazole macrocycles in anion sensors. Beilstein J. Org. Chem. 16, 1901-1914 (2020).

10 Jurček, O. et al. Anion Recognition by a Bioactive Diureidodecalin Anionophore: Solid-State, Solution, and Computational Studies. Chem. Eur. J. 24, 8178-8185 (2018).

11 Marcus, Y. in lons in Solution and their Solvation 107-155 (Wiley, 2015).

12 Tatulian, S. A. Binding of alkaline-earth metal cations and some anions to hosphatidylcholine liposomes. Eur. J. Biochem. 170, 413-420 (1987).

13 Rydall, J. R. \& Macdonald, P. M. Investigation of anion binding to neutral lipid membranes using deuterium NMR. Biochemistry 31, 1092-1099 (1992).

14 Keymeulen, F. et al. Fluoride binding in water with the use of micellar nanodevices based on salophen complexes. Org. Biomol. Chem. 13, 2437-2443 (2015). 
15 Kubik, S. Anion Recognition in Aqueous Media by Cyclopeptides and Other Synthetic Receptors. Acc. Chem. Res. 50, 2870-2878 (2017).

16 Sommer, F., Marcus, Y. \& Kubik, S. Effects of Solvent Properties on the Anion Binding of Neutral Water-Soluble Bis(cyclopeptides) in Water and Aqueous Solvent Mixtures. ACS Omega 2, 3669-3680 (2017).

17 Murgia, S., Monduzzi, M. \& Palazzo, G. Quantification of Specific Anion Binding to Non-lonic Triton X-100 Micelles. Langmuir 28, 1283-1289 (2012).

18 Drummond, C. J., Warr, G. G., Grieser, F., Ninham, B. W. \& Evans, D. F. Surface properties and micellar interfacial microenvironment of n-dodecyl .beta.-D-maltoside. J. Phys. Chem. 89, 2103-2109 (1985).

19 Wu, X. et al. Voltage-Switchable $\mathrm{HCl}$ Transport Enabled by Lipid Headgroup-Transporter Interactions. Angew. Chem. Int. Ed. 58, 15142-15147 (2019).

20 Nymeyer, H. \& Zhou, H.-X. A Method to Determine Dielectric Constants in Nonhomogeneous Systems: Application to Biological Membranes. Biophys. J. 94, 1185-1193 (2008).

21 Stern, H. A. \& Feller, S. E. Calculation of the dielectric permittivity profile for a nonuniform system: Application to a lipid bilayer simulation. J. Chem. Phys. 118, 3401-3412 (2003).

22 Liu, Y., Sengupta, A., Raghavachari, K. \& Flood, A. H. Anion Binding in Solution: Beyond the Electrostatic Regime. Chem 3, 411-427 (2017).

23 Kondo, M., Mehiri, M. \& Regen, S. L. Viewing Membrane-Bound Molecular Umbrellas by Parallax Analyses. J. Am. Chem. Soc. 130, 13771-13777 (2008).

24 Klasczyk, B., Knecht, V., Lipowsky, R. \& Dimova, R. Interactions of Alkali Metal Chlorides with Phosphatidylcholine Vesicles. Langmuir 26, 18951-18958 (2010).

25 Meers, P. \& Feigenson, G. W. Location and ion-binding of membrane-associated valinomycin, a proton nuclear magnetic resonance study. Biochim. Biophys. Acta 938, 469-482 (1988).

26 Tong, Y., Zhang, I. Y. \& Campen, R. K. Experimentally quantifying anion polarizability at the air/water interface. Nat. Commun. 9, 1313 (2018).

27 Jungwirth, P. \& Tobias, D. J. Ions at the Air/Water Interface. J. Phy. Chem. B 106, 6361-6373 (2002).

28 Matile, S. \& Sakai, N. in Analytical Methods in Supramolecular Chemistry (ed Christoph A. Schalley) 711-742 (Wiley-VCH, 2012).

29 Läuger, P. Carrier-mediated ion transport: electrical relaxation experiments give insight into the kinetics of ion transport through artificial lipid membrane. Science 178, 24-30 (1972).

$30 \mathrm{Wu}, \mathrm{X} . \&$ Gale, P. A. Measuring anion transport selectivity: a cautionary tale. Chem. Commun. 57, 3979-3982 (2021). 